\title{
Understanding metro station usage using Closed Circuit TeleVision cameras analysis
}

\author{
C. Carincotte*, X. Naturel ${ }^{\dagger}$, M. Hick* ${ }^{*}$ J.-M. Odobez ${ }^{\dagger}$, J. Yao ${ }^{\dagger}$, A. Bastide ${ }^{\star}$ and B. Corbucci ${ }^{\ddagger}$
}

\begin{abstract}
In this paper, we propose to show how video data available in standard CCTV transportation systems can represent a useful source of information for transportation infrastructure management, optimization and planning if adequately analyzed (e.g. to facilitate equipment usage understanding, to ease diagnostic and planning for system managers). More precisely, we present two algorithms allowing to estimate the number of people in a camera view and to measure the platform time-occupancy by trains. A statistical analysis of the results of each algorithm provide interesting insights regarding station usage. It is also shown that combining information from the algorithms in different views provide a finer understanding of the station usage. An end-user point of view confirms the interest of the proposed analysis.
\end{abstract}

\section{INTRODUCTION}

Despite the legitimacy of a number of privacy issues, Closed Circuit TeleVision (CCTV) networks are nowadays commonly present in public environments such as transportation premises, city centers or commercial establishments. In the meantime, automatic processing of video data is currently a field of activity stirring up the utmost attention in the pattern recognition community; state-of-the-art advances in this area enable the reliable extraction of surveillancelike events such as person tracking, face/object recognition, abnormal behavior and abandoned luggage detection.

Apart from surveillance and safety issues, CCTV video streams may also represent a useful source of information for urban planning and resource optimization applications. Advanced video analysis devices can indeed provide above physical sensors information and wide-area measurements that can replace, or at least complement, many conventional physical detectors. In addition, video detection performance can be easily verified and detectors are easy to reconfigure interactively. In this context, very few works address the issue of using already deployed CCTV network to perform statistics gathering (relatively unexplored problem), e.g. for maintenance/planning purposes (people counting, person classification, speed measurement...). Indeed, most studies deal with surveillance-like event detection and scenario

The work presented here is partially supported by the European Commission under the 6th Framework Program through the IST FP6-027231 CARETAKER project. For further information about the CARETAKER project, please visit http://www.ist-caretaker.org/.

* MUltitel asbl, Rue Pierre et Marie Curie 2, 7000 Mons, Belgium \{carincotte, hick\}@multitel.be

† IDIAP Research Institute, Rue Marconi 19, 1920 Martigny, Switzerland \{naturel, odobez, yao\}@idiap.ch

* ACIC s.a./n.v., Rue René Descartes 2, 7000 Mons, Belgium bastide@acic-tech.be

$\ddagger$ ATAC SpA, Via Ostiense, 131L, 00154 Roma, Italy bruno.corbuccieatac.roma.it recognition [1], allowing for example to determine whether a human is crossing the rails [2], [3], to detect overcrowding situation in the platform [4], or to produce an aggression indication [5].

In this context, our contribution is threefold. We first propose two algorithms, one to estimate the number of people in one camera view, and the other to measure the platform timeoccupancy by trains. The second contribution is to provide a statistical analysis on a large dataset of the results of these detectors, to provide a better understanding of transportation stations usage from the planning and resource optimization point of view. The goal of this statistical analysis is to provide long term analysis of the station's usage, through patterns of activity discovery, and detection of abnormal events. Eventually, the joint analysis of different camera views allows to provide a better and more complete analysis of station's usage, for instance in analysing whether too much people on the platform delay the departure of the train.

The structure of the article is as follows. Sec. II explains the algorithms for detecting people and train arrivals/departures. Sec. III provides an analysis on the results of each algorithm. Sec. IV then uses the combined results to provide a joint analysis. Finally, feedbacks on the practical interest of such an analysis from the user point of view are given in Sec. V.

\section{VIDEO DETECTORS}

In this section, we provide a brief theoretical description of the algorithms used to perform people detection and platform occupancy measure.

\section{A. Human detection}

We briefly present in this section the fast method we developed to detect humans in videos captured in surveillance applications. Interested readers may consult [6] for more details.

The proposed algorithm is based on a cascade of LogitBoost classifiers relying on features mapped from the Riemanian manifold of region covariance matrices computed from input image features. The developed human detector relies on the approach of Tuzel et al. [7], which was shown to provide good detection performance for human detection in still images. It was improved by extending in several ways [6]. First, as the mapping process is slow for high dimensional feature space, we propose to select weak classifiers based on subsets of the complete image feature space. In addition, we propose to combine these sub-matrix covariance features with the means of the image features 
computed within the same sub-window, which are readily available from the covariance extraction process. Finally, in the context of video acquired with stationary cameras, we propose to fuse image features from the spatial and temporal domains in order to jointly learn the correlation between appearance and foreground information based on background subtraction. Our developed method can process from 5 to 20 frames/sec (for a $384 \times 288$ video), while achieving similar or better performance than existing methods.

\section{B. Train arrival/departure detection}

The proposed method ${ }^{1}$ is mainly based on a tracking algorithm, which proved to be efficient in various contexts (indoor/outdoor, metro/train, camera location...). This approach does not use any background modeling estimation, thus preventing from context changes related issues, such as illumination, reflection... The main idea of the approach is to use trajectories from randomly distributed particles in the image to perform the train arrival/departure detection (and corresponding platform occupancy computation). Next paragraphs give an overview of the algorithm principle and stages.

The principle of the proposed method is to quickly locate moving objects in the scene, and to determine whether their motions are compatible with the requirements of the train arrival/departure (location, direction, speed...). To do so, particles are randomly initialized in a region of interest (i.e. "rails zone"), and tracking is activated for each particle when a defined criterion is met (roughly when motion is detected). Relevant trajectories can then be analyzed to compute useful information and eventually derive the final train arrival or departure decision.

Particle distribution: Inactive particles are randomly distributed for each new image with a non-uniform rule using the calibration information, to take into account perspective over the region of interest (detection area).

Particle activation: The activation criterion is based on an instantaneous motion detection, namely a thresholded frame-differencing operator. When an inactive particle is moved to a point where the frame-differencing operator is bigger than a defined threshold, the tracking for the concerned particle begins.

Particle tracking: After activation, a particle continuously tracks the motion of the underlying object using a block-matching algorithm. Particles associated with uninteresting trajectories are recycled as detailed below.

Filtering of trajectories: Trajectories are analyzed by computing a set of various features; linearity of track, track length, track duration, track direction, start/stop particle location... Trajectories are then classified as relevant ones and uninteresting ones depending on the features' values. An uninteresting trajectory is then recycled while a relevant one is kept active. Typically, trains/metros are characterized by well-defined trajectories, i.e. linear trajectories, mean direction parallel to the rails, speed linearly

\footnotetext{
${ }^{1}$ Intelligent Video System Software, Copyright (C)2008 ACIC. For further information, please visit http://www.acic.eu/.
}

increasing/decreasing... On the other hand, passengers and tracking errors are most likely to have much more chaotic trajectories, which make them rejected.

Train arrival/departure detection: All remaining trajectories are then scored; low values are attributed to trajectories of weak interest, while high scores are given to highly relevant ones. The total score is compared to a threshold to decide whether a train is potentially arriving/departing; arrival/departure time are lastly estimated using a Finite State Machine (FSM), which allows to distinguish between arrival and departure, and to compute the resulting platform timeoccupancy.

\section{SINGLE VIEW ANALYSIS}

In this section, both video detectors are applied on large amounts of data; the detection results are then evaluated and analyzed on an individual basis.

\section{A. Data description}

Experiments were conducted using a dataset acquired during the CARETAKER project [8]; videos come from 14 acquisition sessions performed in Roma metro on June 2007 (all sessions were performed from 06.00 a.m. to 11.30 a.m.). The resolution of all sequences are PAL standard $(720 \times 576$ pixels, 5 frames per second) and compressed using MPEG- 4 . Two cameras are used for the experiments, one monitoring the platform (Fig. 1-(b), called "platform view") and one monitoring the turnstiles to access the train platform (Fig. 1(a), called "turnstiles view"). In this view, several flows of people are mixing up; people can come from the left or from the top and go through the turnstiles to access the train platform, people can inquire at the desk (middle top of the image), and people leaving the platform can also be seen at the bottom of the image. A small part of the platform can also be seen on the top right of this view.

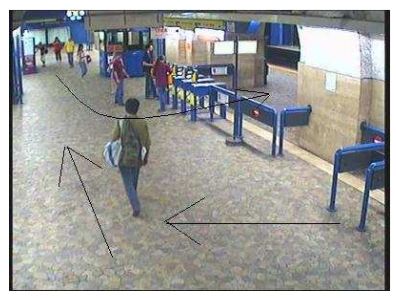

(a) "Turnstiles view".

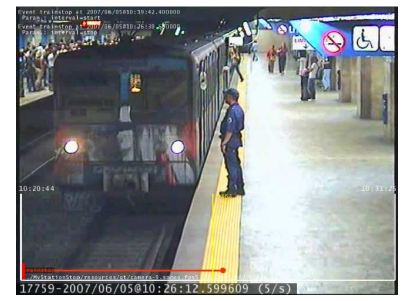

(b) "Platform view".
Fig. 1. Camera views used for experiments.

\section{B. Monitoring activity in turnstiles view}

In a first step, we estimate the number of people over time in the turnstiles view (Fig. 1-(a)). The human detector of Sec. II-A is thus applied every second of the video (i.e. every 5 frames). As a matter of fact, the same person can be counted several times in the video stream. This measure cannot therefore be considered as an estimation of the number of unique people passing through the station, but has to be interpreted as a measure of scene/space occupation at each time instant. A post-processing step is used to smooth 
the detection output, by averaging the number of people on a 3 minutes window.

Fig. 2 shows this smoothed signal, where each point is thus the average number of people on a $3 \mathrm{~min}$ window. In the rest of the paper, if not clearly mentioned, the detection results presented will correspond to this smoothed signal.

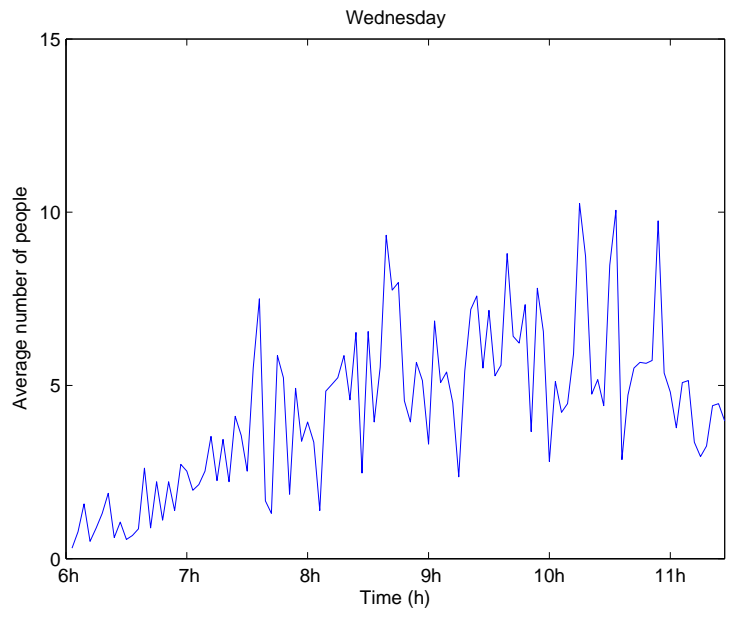

Fig. 2. Average Number of people (on a 3 min window) over time in turnstiles view (Wednesday morning).

Using the (smoothed) detection results for all sequences (i.e. 14 consecutive mornings), an analysis is conducted to extract and visualize the global trend of activity, and see how the evolution over one morning fits into the average trend.

While analyzing these two weeks of data, we specifically distinguish between week days and week-ends, to see if week-ends stand out from week days. To do so, the average and standard deviation over week days are computed at each time instant, e.g. with $c_{k}(t)$ the smoothed number of counts for morning $k$ at time $t$ and $N=10$ the number of mornings, we compute $m(t)=\frac{1}{N} \sum_{k=1}^{N} c_{k}(t)$ and $\sigma^{2}(t)=\frac{1}{N-1} \sum_{k=1}^{N}\left(c_{k}(t)-m(t)\right)^{2}$.

Fig. 3-(a) shows the week average $m(t)$ (in green), as well as its $\mathrm{fit}^{2}$ (blue), and the fit $\pm 2 \times \sigma(t)$ (red) ${ }^{3}$. These curves characterize the usual usage of the station on week days, and the envelope of 2 standard deviations (red curves) indicates the area where the behavior can be considered as "normal". A simple analysis shows that the average usage is more or less what we could expect: traffic is low from 6.00 am to $7.30 \mathrm{am}$; it then increases steadily until 9.30 am and slowly decreases starting from 10.30 am. On Fig. 3-(b) is displayed an example of how a random week-day (here a Wednesday) fits into this week average, and how close its evolution (in magenta on the figure) is from the week evolution.

Week ends are analyzed in Fig. 4, where the average of the 2 Saturdays and Sundays is plotted together with the week average. It allows to show the difference in evolution of the

\footnotetext{
${ }^{2}$ The fit is computed using a smoothing spline curve-fitting function, and a smoothing parameter of 0.0003 .

${ }^{3}$ We remind that for a gaussian distribution of the observations, $95 \%$ of the observations fall within 2 standard deviations of the mean, i.e. between $m-2 \sigma$ and $m+2 \sigma$.
}

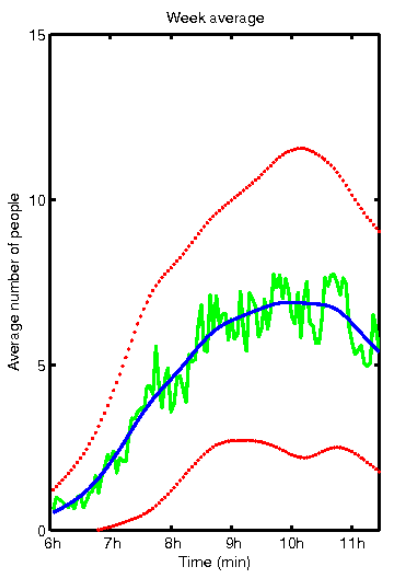

(a) Week-days average.

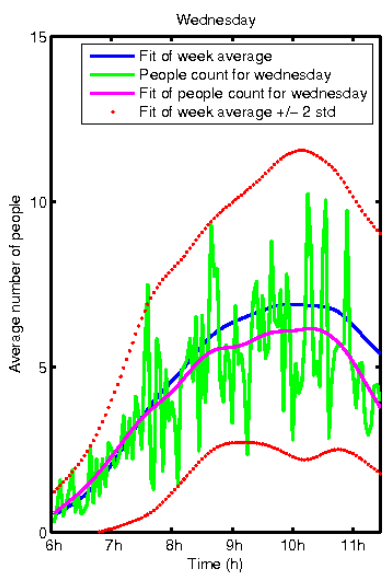

(b) Wednesday vs week-days average.
Fig. 3. Plots of average people density (plus fit and standard deviation) for week days and Wednesday.

number of people on Saturday and Sunday mornings, and especially that it does not fit into the week average curves. Saturday appears to be a busy day; there is an early start and it also does not comply with the decrease around 10.30 am observed on week days. On the other hand Sunday is clearly less busy, and if the curve has approximately the same shape as the week days one, it is clearly shifted to the right, indicating that people are getting up late, as one might have expected.

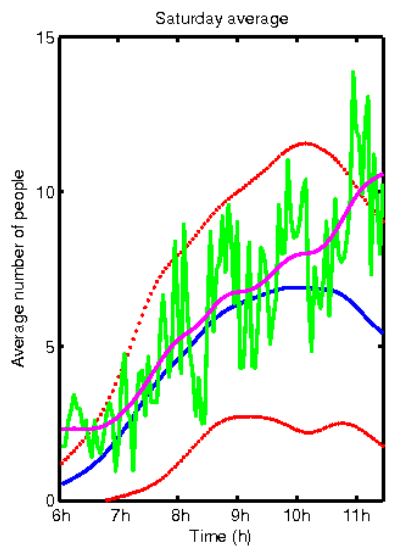

(a) Saturdays average.

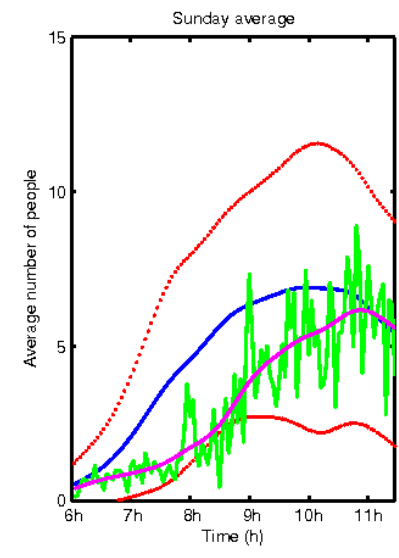

(b) Sundays average.
Fig. 4. Saturday and Sunday averages (magenta), compared to the week average (blue).

As highlighted in Fig. 5, the behavior observed in Fig. 3 (Wednesdays average) can be extended to the whole weekdays sequences of the dataset. As clearly shown in Fig. 5-(a), the behavior observed for Wednesdays is quite similar to the one observed for the other week-days, for which all plots are located around the average plot. As for the week-end days (Fig. 5-(b)), the two Saturdays seem to have very different scene occupations, and are therefore difficult to analyze in a proper way; such behaviors will be more analyzed in the 
following sections. Regarding Sundays, both plots are clearly similar, and exhibit a later start of activities within the station, which confirms the conclusions initially drawn.

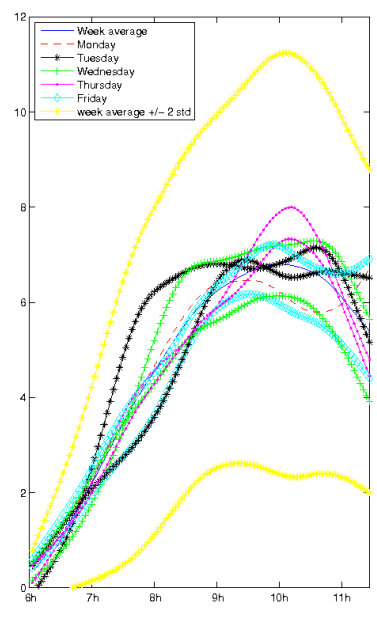

(a) Day by day fits

of week-days average.

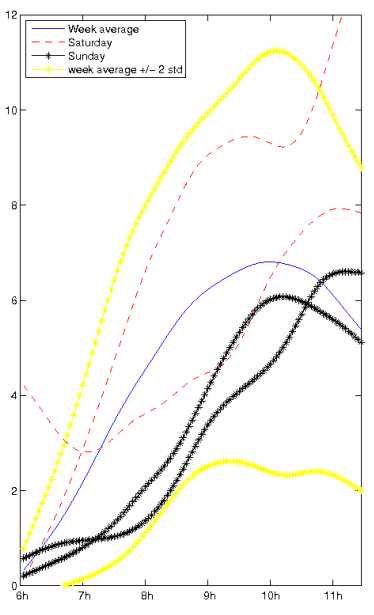

(b) Fits of Saturdays and Sundays average.
Fig. 5. Fit of all week-days and week-ends days average, compared to the week average (blue).

Eventually, Fig. 6-(a) shows an interesting example on a week day (Thursday). While the global trend follows the week average, an unusual event stands out well of the 2 standard deviation envelope (around 10.00 a.m.). This peak is due to a group of obviously lost tourists, which is staying still or wandering about, for quite a long time, moving in and out of the camera view (see Fig. 6-(b)).

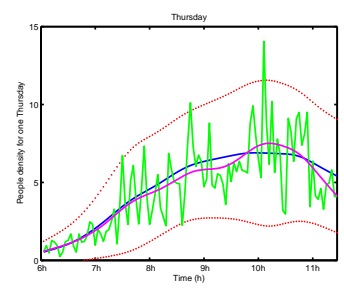

(a) People density on one Thursday.

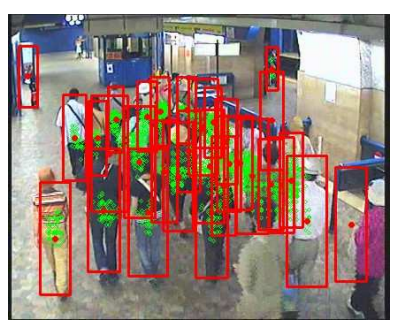

(b) Group of tourists causing peak in (a).
Fig. 6. People density on one Thursday, compared to the week average (a). An unusual event (group of lost tourists, staying still or wandering about (b)) can be spotted around 10.00 a.m.

\section{Monitoring train traffic}

In this section, we first evaluate the time-occupancy measure presented in Sec. II-B. We then analyze the detection results to identify general trends in the metro operation.

The accuracy of the train stop estimation was firstly measured using annotated data (ground truth), performed manually on the whole 14 sequences. Tab. I shows the detection rates obtained during the evaluation process. As highlighted in this table, the average detection rate on the overall sequences is around $96.59 \%$ (710 detections on 735 stops in the GT), which demonstrates the effectiveness and robustness of the proposed approach.
Regarding the detection delays at train arrival/departure, most of them are between $0.5 s$ and $1 s$ which seems to be negligible regarding the stop-duration itself (average value of $45 \mathrm{~s}$ on the overall sequences). As illustrated in Fig. 7 which presents a detection result on a single sequence, the arrival/departure missed-detections or false-alarms (respectively positive and negative delays) are almost insignificant with respect to the detection duration.

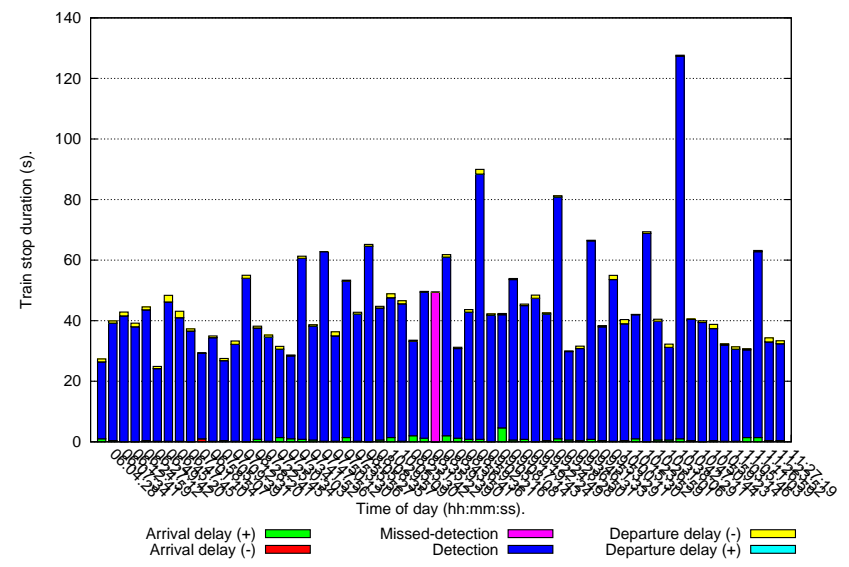

Fig. 7. Detection results (stacked histogram) obtained on sequence "Morning 2007/06/04".

When computing train stop statistics on a sequence basis (e.g. by computing an average train stop value by sequence, and its corresponding standard deviation, see Tab. I), we can observe several interesting points. The estimated average trainstop duration is very closed to the ground truth (GT) one; this firstly confirms the effectiveness of the proposed approach. Both GT and estimated values also show that the time-occupancy of the platform can have very different durations depending on the concerned day; e.g. week-days seems to have longer average occupation than week-end days. In a second time, standard deviations (for both GT and estimated values) also reveal interesting behaviors. While most of standard deviation (std) values are quite closed to (or little higher than) what could have been expected, some of them seem to be very high in comparison to their respective mean values (e.g. see Sunday 07.06.03, Monday 07.06.11, and especially Tuesday 07.06.05 in Tab. I).

So as to understand such phenomena, Fig. 8 shows the histogram of the train occupancy-time in the station computed on the whole sequences, for both GT and processed data. It shows that the time-occupancy of the platform can have very different durations; while the bulk of the detections are located around 30-50s (average value of $45 \mathrm{~s}$ ), a nonnegligible number of stops are far below (10s) or far above (up to $3 \mathrm{~min}$, even $6 \mathrm{~min}$ ).

In the transportation context, such outlying values can be explained by several phenomena; regulation purposes, safety checks, incidents on platform, signal failures or breakdown of systems... In our case, after inspecting the related timeslots in the videos, most of these outliers come from regulation purposes and incidents on the platform. For example, Fig. 9-(a) shows people trying to enter in the metro 
TABLE I

PLATFORM TIME-OCCUPANCY BY METROS: DETECTION RESULTS

\begin{tabular}{|c|c|c|c|c|c|c|c|}
\hline Day & 07.06.02 (Sat) & 07.06 .03 (Sun) & 07.06.04 (Mon) & 07.06.05 (Tue) & 07.06.06 (Wed) & 07.06.07 (Thu) & 07.06.08 (Fri) \\
\hline Detection rate (time) & $100 \%$ & $95.90 \%$ & $98.20 \%$ & $97.89 \%$ & $97.05 \%$ & $100 \%$ & $91.61 \%$ \\
\hline Mean arrival delay (s) & 0.77 & 0.63 & 0.58 & 0.83 & 0.63 & 0.78 & 0.62 \\
\hline $\begin{array}{l}\text { Average stop duration (std) } \\
\text { - detection - } \\
\text { - }\end{array}$ & $37.14(10.53)$ & $\begin{array}{l}42.77(35.73) \\
43.21(35.40)\end{array}$ & $\begin{array}{l}44.41(16.86) \\
44.34(16.84)\end{array}$ & $\begin{array}{l}53.98(52.92) \\
53.22(52.21)\end{array}$ & $\begin{array}{l}46.63(15.87) \\
46.08(15.98)\end{array}$ & $\begin{array}{l}45.70(29.74) \\
45.99(29.66)\end{array}$ & $\begin{array}{l}45.33(22.58) \\
45.38\end{array}$ \\
\hline
\end{tabular}

\begin{tabular}{|c|c|c|c|c|c|c|c|}
\hline Day & 07.06 .09 (Sat) & 07.06 .10 (Sun) & $07.06 .11($ Mon) & $07.06 .12($ Tue) & $07.06 .13($ Wed) & $07.06 .14(\mathrm{Thu})$ & $07.06 .15(\mathrm{Fri})$ \\
\hline Detection rate (nb) & $98.03 \%$ & $\begin{array}{c}97.22 \% \\
(50 / 51)\end{array}$ & $\begin{array}{c}98.18 \% \\
(35 / 36)\end{array}$ & $\begin{array}{c}98.18 \% \\
(54 / 55)\end{array}$ & $\begin{array}{c}94 \% \\
(47 / 50)\end{array}$ & $\begin{array}{c}91.80 \% \\
(56 / 61)\end{array}$ & $\begin{array}{c}96.66 \% \\
(58 / 60)\end{array}$ \\
\hline Detection rate (time) & $97.86 \%$ & $97.58 \%$ & $97.79 \%$ & $98.16 \%$ & $95.05 \%$ & $92.91 \%$ & $96.04 \%$ \\
\hline Mean arrival delay (s) & 0.72 & 0.41 & 0.71 & 0.68 & 0.98 & 0.76 & 0.00 \\
\hline Mean departure delay (s) & 0.82 & 0.60 & 0.73 & 0.85 & 1.11 & 0.71 & 0.73 \\
\hline Average stop duration (std) & & & & & & & \\
- detection - & $35.13(15.08)$ & $38.96(22.73)$ & $48.37(32.60)$ & $48.95(17.59)$ & $51.04(30.45)$ & $42.19(15.31)$ & $43.73(25.76)$ \\
- ground truth - & $35.09(14.91)$ & $38.62(22.22)$ & $48.54(32.41)$ & $48.79(17.51)$ & $50.35(29.82)$ & $41.74(15.12)$ & $43.27(25.24)$ \\
\hline
\end{tabular}

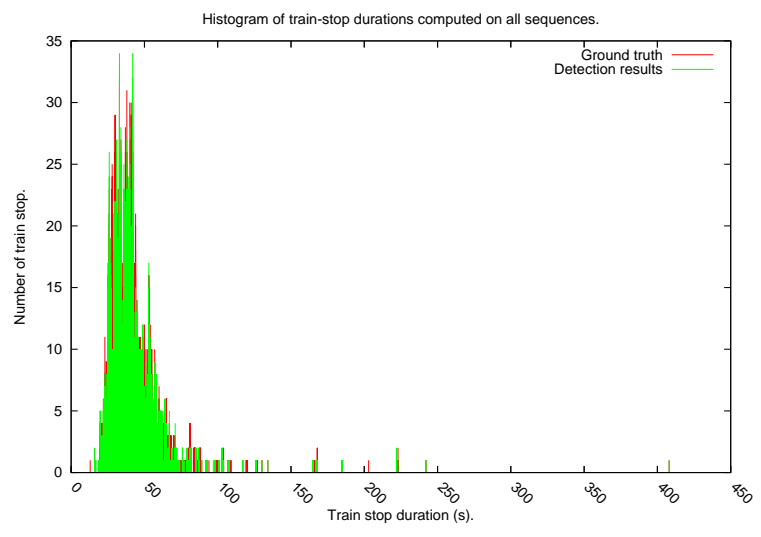

Fig. 8. Histograms of trainstop detection computed on the 14 sequences.

with a quad, and being intercepted by policemens (trainstop of 100s), while Fig. 9-(b) corresponds to regulation purpose (trainstop of 408s).

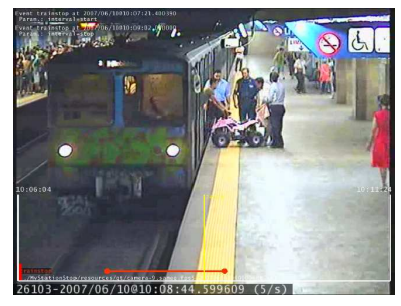

(a) $2007 / 06 / 10-100 \mathrm{~s}$

"quad intrusion". (b) $2007 / 06 / 05-408 \mathrm{~s}$

"regulation purpose".

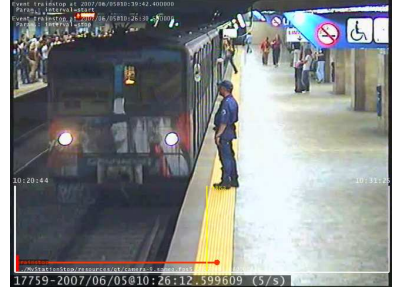

Fig. 9. Screenshots corresponding to trainstop duration outliers.

From the metro operation point of view, Tab. I also allows to identify different trends depending on the days; e.g. weekend days seems to have both less metros and lower average detection than week-days. So as to confirm this observation, Fig. 10 presents cumulative plots of platform occupation for each sequence; such graph allows to reflect both trainstop duration and frequency within a single plot. It clearly exhibits the fact that Saturdays and Sundays have lower platform occupation compared to the other days of the week. This

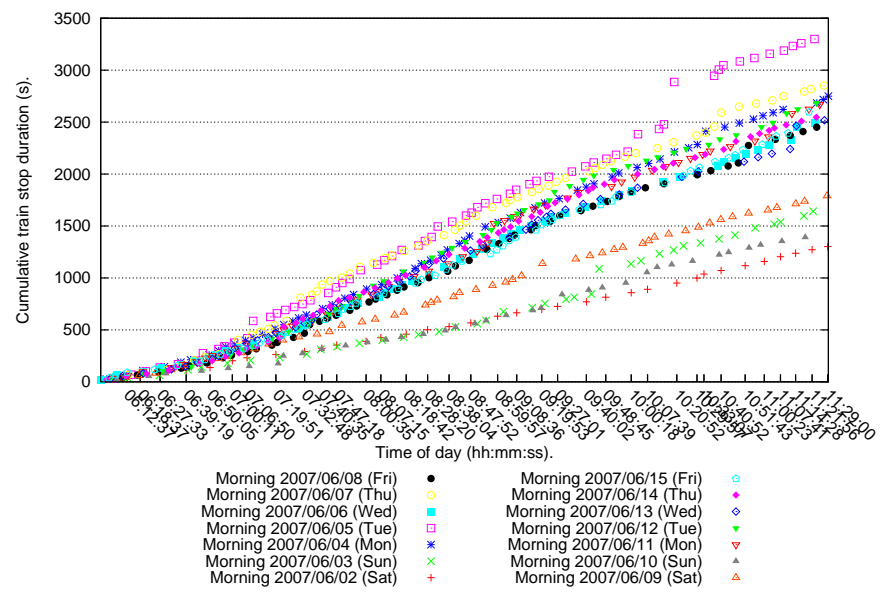

Fig. 10. Plot of cumulative platform occupancy for all sequences.

conclusion is different from the one obtained in Sec. III-B for Saturdays, where we observed in average more people in the turnstiles view. The next section will investigate the joint interpretation of the two views outputs, and especially address this apparent contradictory issue.

Eventually, so as to identify general trends in the weekly operation of the metro, we propose to approximate the data with a Bezier curve of degree $n$ ( $n$ being the number of stops in the video sequence). Fig. 11 presents corresponding plots, where outliers (here considered as trains that stop more than $100 s$ ) are discarded.

Fig. 11 exhibits the fact that all week-day plots seem to be linear piecewise. More precisely, the week-day plots may be approximated with three straight lines, for three different ranges of hours (delimited in Fig. 11 by the two vertical dotted lines). Such possible piecewise linear approximation characterizes the varying distribution of metros along the morning; indeed, metros are much more present from 07.00 to $\sim 09.45$ a.m. than from 06.00 to $\sim 07.00$ a.m.. On 


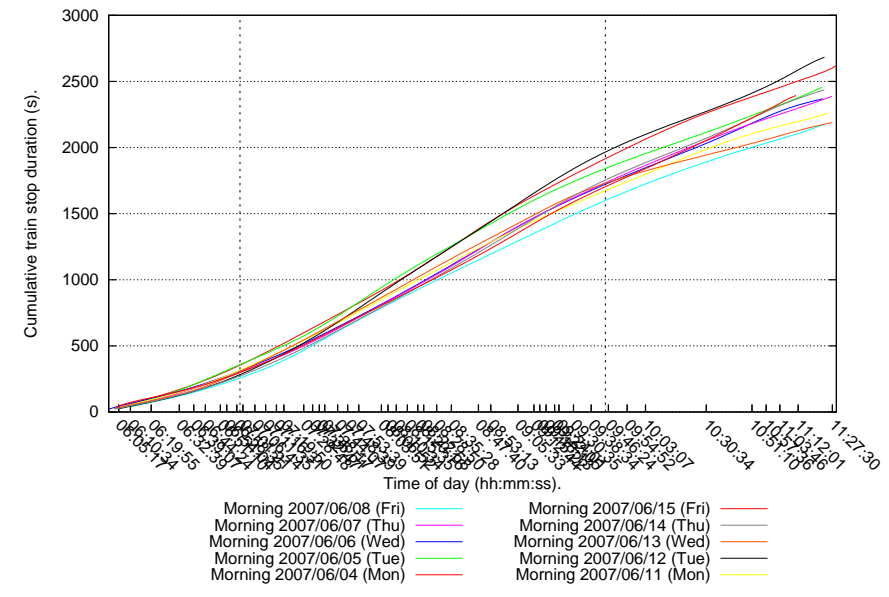

Fig. 11. Plot of smoothed cumulative platform occupancy for week-days sequences (outliers are discarded).

the other hand, metros' distribution from $\sim 09.45$ to 11.30 a.m. is a trade-off between the two previous hours ranges. Such results are quite consistent with the ones obtained in Sec.III-B (especially Fig. 3-(a) which characterizes the weekly station usage). As expected, week-days traffic (in both people and metros) is low in the early morning, then quite higher for office hours start, and then decreases.

Fig. 12 presents similar Bezier approximation plots for week-end days (outliers are also discarded). While these plots are interesting, the analysis of the results has to be performed carefully since only few (and maybe not sufficiently representative) data were available for these days (2 sequences for each figure in comparison to 10 sequences for Fig. 11). Indeed, even if the two Saturdays analyzed seem to be quite different in terms of values, both plots in Fig. 12(a) exhibit a ramp up behavior. This tends to suggest that Saturdays operation is quite linear, i.e. without no distinct stages in the metro operation. Regarding Sundays operation, Fig. 12-(b) suggests that plots are piecewise linear, for two different ranges of hours. While the exact ranges are quite difficult to estimate with only two plots, it already confirms the observations made in Sec. III-B, i.e. that activities in the station on Sundays start later than on week-days.

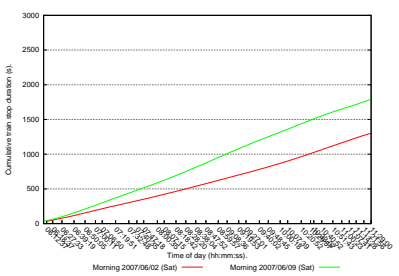

(a) Saturdays smoothed plots.

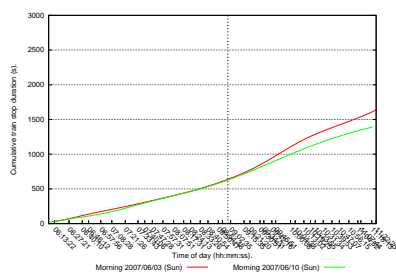

(b) Sundays smoothed plots.
Fig. 12. Plot of smoothed cumulative platform occupancy for week-end days sequences (outliers are discarded).

So as to benefit from the two analysis performed in this section, next section proposes a joint analysis of the results obtained on the two cameras' views.

\section{MULTI-VIEW ANALYSIS}

In this section, we investigate the joint usage of information coming from the two camera views, to provide a more in-depth analysis and solve possible ambiguities.

\section{A. Understanding peaks of activities}

We are looking at the relation between the number of people in the turnstiles view and the trainstop measure, using results from Sec. III-C and III-B.

Fig. 13 shows an example of 9 train arrivals and departures over one hour, with the corresponding number of people in the turnstiles view. In this case, the signal is not averaged, i.e. the values displayed are the actual number of people at each second. It shows quite clearly that the peaks of the number of people in the turnstiles view correspond to instants where the train is present in the station. These peaks are due to people leaving the train, and passing in front of the turnstiles camera view, towards the exit. On average, it was computed that there are 7.2 persons in the turnstiles view when a train is present, whereas there are only 4.5 persons in the other case.

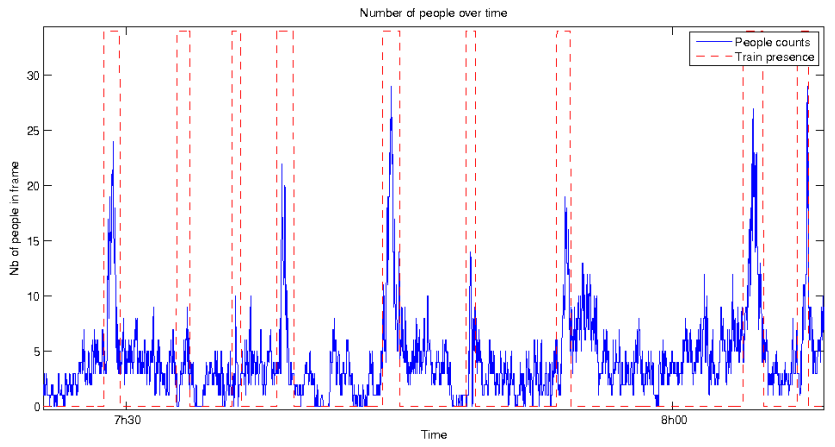

Fig. 13. Overlay of train arrivals and corresponding counts of people.

\section{B. Understanding flows of persons}

Another useful way of using the train presence information is to make the same analysis as in Fig. 3, with filtering out the instants where the train is present. More precisely, the number of people in the time slot where the train is present is replaced by the average in the period where no train is in the platform (e.g. when no flow of people is coming out of the platform). This filters out from the signal the peaks due to people coming out from the train. The obtained signal thus characterizes the usual activity in the turnstiles view, excluding the arrivals. Fig. 14 shows this filtered signal and its fit (in magenta), compared with the fit of the non-filtered signal (in black). From this figure, it seems that the high variability in the number of people in the turnstiles view is not only due to train arrivals. This means that independently from train arrivals, the activity in the turnstiles view is itself very irregular. This is confirmed by the computation of the average standard deviation, which is 3.1 for the non-filtered people density signal and 2.6 for the filtered one, which is still quite high. 


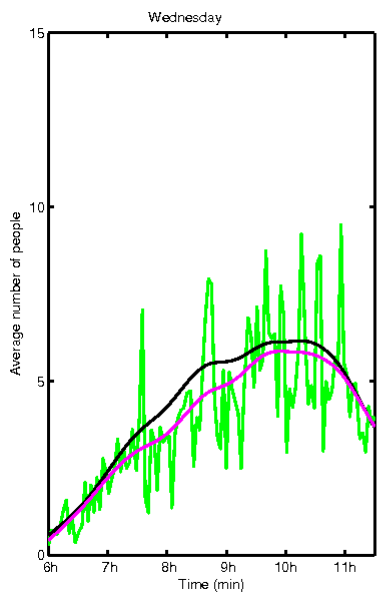

(a) Wednesday.

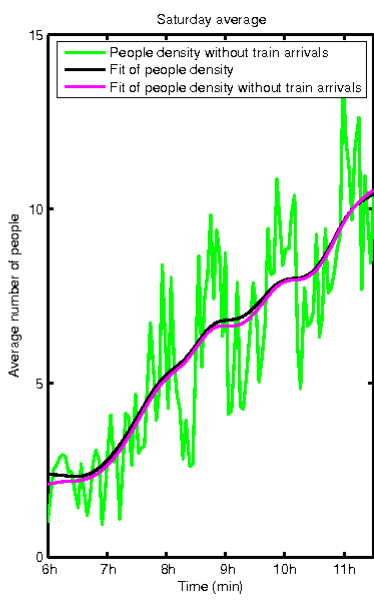

(b) Saturday.
Fig. 14. People density and its fit, without and with filtering out train arrivals ((a) Wednesday and (b) Saturday).

Another important remark is that the difference between filtered and non-filtered signals is quite noticeable for week days (one Wednesday is shown on Fig. 14-(a)) whereas it is barely noticeable for Saturdays and Sundays (Saturdays average is shown in Fig. 14-(b)). This means that far less people are coming out of the trains on week-ends, and thus the traffic in the turnstiles view is mainly due to people passing by or going to the platform. This is confirmed by Fig. 15, which shows the average number of people per morning, on train presence time (blue) and on train absence (green). The difference between the 2 curves is clearly larger on week days (3.1 in average) than on week-ends (1.5 in average). This clearly shows a different behavior of users on week-ends.

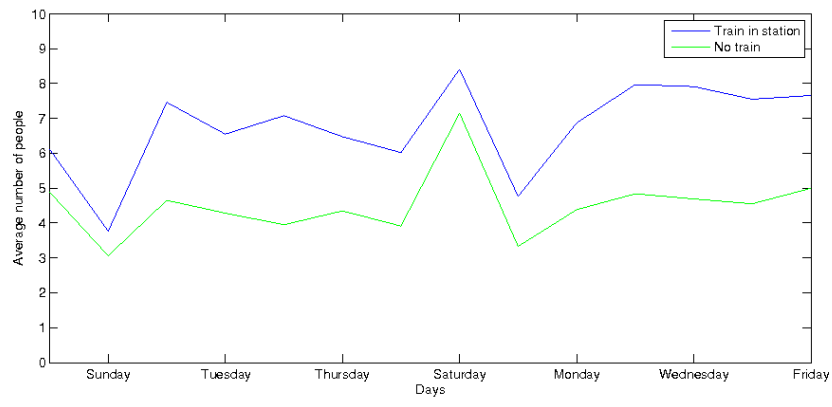

Fig. 15. Average number of people per morning when train present (blue) or not (green).

In particular, the apparent contradictory results for Saturdays (more people in average in turnstiles view but less trains, and feedbacks from Rome operatives that Saturdays are less busy overall) are explained: the high people density is not related to train exits, but is due to people waiting or entering the platform. Several hypothesis are proposed to explain this high density. Feedbacks from the video hinted a difference of behavior between week days users (mostly people going to work) and week-ends users (occasional travelers), which tend to stay longer in the camera view, waiting or hesitating on the way to go. The second one is that people take different routes depending on the days (weekdays or week-ends), and flows in this camera view are thus different.

Note that this behavior could not have been spotted by the monitoring of the turnstiles view alone. This joint analysis is thus a very useful tool that highlights a difference of behavior and station usage on specific days.

\section{Analysing train staying duration and platform crowding}

One of the interest of the metro operatives is to see whether too much people on the platform delay the departure of the train. We thus try in this section to relate the estimated number of people on the platform with the train staying duration. We are measuring the number of people in the turnstiles view (excluding instants where people leave, i.e. train present in station), which is just one entry point to the platform, the goal is thus more on catching the trend than on the exact estimation of the number of people.

To verify this hypothesis, the correlation between the train staying duration and the cumulative number of people in the turnstiles view (excluding train arrivals) is computed. The empirical correlaton coefficient is given by

$$
r=\frac{1}{N-1} \sum_{k=1}^{N} \frac{\left(x_{k}-\bar{x}\right)\left(y_{k}-\bar{y}\right)}{\sigma_{x} \sigma_{y}}
$$

where $N$ is the number of samples, and $\sigma$ are the empirical standard deviations. Outliers, e.g. trains that stay more than 100 s (2 times the maximum standard deviation of stop durations) are discarded. Results in Tab. II show that most

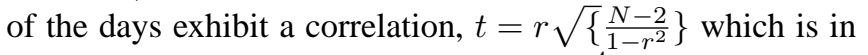
most of the cases above the critical value ${ }^{4}$, under which we cannot reject the null hypothesis (e.g. the variable are not correlated). Exceptions are Sundays, where the correlation does not seem to be significant.

Fig. 16 shows the relation between the average number of people during waiting time (time between a train leaves and another arrives) and the time that the next train stays in station. It also confirms the results of Tab.II, that there seems to be a link between the two variables, but which cannot explain the outliers. The platform crowding level can thus be an explanation for some delay and the train irregularity, but cannot explain the observed variations.

\section{END-USER FEEDBACKS}

CCTV has been traditionally installed in underground environment to support the operation management with a view to enhance safety and security. Nowadays modern advance video processing technologies are opening a new role for CCTV. As CARETAKER project [8] is demonstrating, new algorithms can provide valuable information to underground and public transport operations and security managers. The possibility to automatically process on-line and massive recorded data, within the respect of privacy regulation, can

\footnotetext{
${ }^{4}$ Critical values are extracted for a $5 \%$ risk, from tables in [9].
} 
TABLE II

CORRELATION BETWEEN TRAIN STAYING DURATION AND CUMULATIVE NUMBER OF PEOPLE IN TURNSTILES VIEW.

\begin{tabular}{|c|c|c|c|c|c|c|c|c|c|c|c|c|c|c|}
\hline Days & Mon & Tue & Med & Thu & Fri & Sat & Sun & Mon & Tue & Wed & Thu & Fri & Sat & Sun \\
\hline filtering outliers Correlation & 0.75 & 0.43 & 0.77 & 0.40 & 0.60 & 0.44 & 0.31 & 0.58 & 0.44 & 0.76 & 0.20 & 0.57 & 0.51 & 0.31 \\
\hline Critical value & 0.25 & 0.25 & 0.28 & 0.26 & 0.27 & 0.33 & 0.27 & 0.27 & 0.25 & 0.27 & 0.25 & 0.25 & 0.32 & 0.32 \\
\hline
\end{tabular}

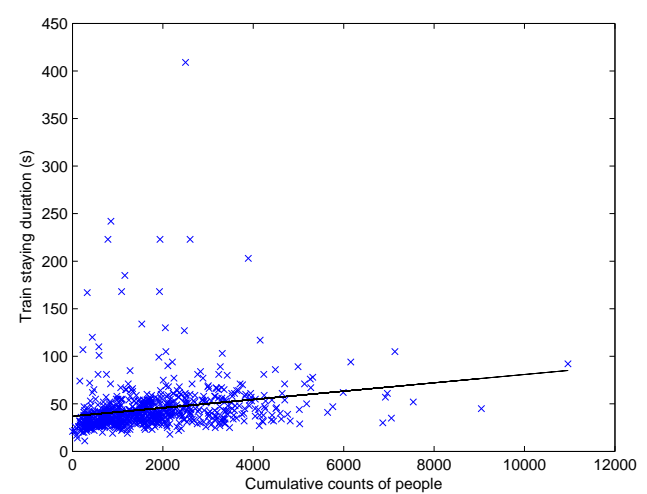

Fig. 16. Relation between cumulative number of people in turnstiles view, and time spent by the train in station

indeed help the security staff which can not have possibility to process such data.

The algorithms described in this paper fulfill such requirements. The estimation of number of people in a camera view can help to alert the operator when anomalies may occur e.g. when a station reach its capacity limit that request the limitation of the number of people entering the station (in Rome this alert can be risen in some station during large events). As a long-term analysis along with ticketing data fusion, it can help to provide a clear view of the trend of how the station (or its specific part) is used.

The algorithm that analyzes the platform time occupancy by the train can support the operations (that already have the signaling system). It can support the analysis of anomalies, with a direct reference to the related video data, to improve in the longer term the performances and regularity of the service.

The most interesting thing, as shown in the previous section is to combine the analysis of the two algorithms. The in depth analysis of such results could also lead to a redesign of the level of service provided by the underground, taking also into account the transportation demand.

To the best of our knowledge, this is the first attempt to analyze such information, which is very new in the transportation world and needs some time to be integrated in the usual evaluation. Furthermore the estimation of number of people in a camera view could provide more useful information if the analysis is carried out in more cameras and potentially comparing different stations. Also the analysis of the platform time occupancy by the train can provide more valuable information if extended on several stations, to understand the domino effect of potential delays.

\section{CONCLUSION}

A person detector and a train arrivals/departures detector have been used on a large amount of real video data of the Rome underground. It has been shown that a statistical analysis of the results provides interesting information regarding station usage, allowing to characterize the usual behavior of train users, to distinguish trends between days of the week, and spot some unusual events. It has also been shown that the joint usage of the information coming from two views can solve possible ambiguities and can provide a better understanding of the station's usage. As perspectives, this joint analysis will be extended to several views (e.g. all the cameras monitoring the plaform access or even an entire station) and with larger amount of data; such study will allow to confirm the obtained results, and to demonstrate the reliability and the interest of the proposed tools. An extension to the size of a underground network, to better understand the relationships between stations and their usage could also be envisaged.

The exploitation of the potential of such technology, along with the comprehension and the analysis of results, could lead to a new way to exploit CCTV resources in the underground, to enhance both safety and security.

\section{ACKNOWLEDGMENTS}

The authors are grateful to G. Lucisano from Met.Ro. (Metropolitana di Roma s.p.a.) for providing valuable insight and guidance on Roma metro operation.

\section{REFERENCES}

[1] A. Cavallaro, "Event detection in underground stations using multiple heterogeneous surveillance cameras," in Advances in visual computing. ( First international symposium, ISVC 2005), vol. 3804, Lake Tahoe, NV, USA, December 5-7 2005, pp. 535-542.

[2] S. Oh, S. Park, and C. Lee, "A platform surveillance monitoring system using image processing for passenger safety in railway station," in Control, Automation and Systems, 2007. ICCAS '07. International Conference on, Seoul, 17-20 Oct. 2007, pp. 394-398.

[3] - "Vision based platform monitoring system for railway station safety," in Telecommunications, 2007. ITST '07. 7th International Conference on ITS, Sophia Antipolis, 6-8 June 2007, pp. 1-5.

[4] B. Lo and S. Velastin, "Automatic congestion detection system for underground platforms," in Intelligent Multimedia, Video and Speech Processing, 2001. Proceedings of 2001 International Symposium on, Hong Kong, China, 02-04 May 2001, pp. 158-161.

[5] W. Zajdel, J. Krijnders, T. Andringa, and D. Gavrila, "Cassandra: audiovideo sensor fusion for aggression detection," in Advanced Video and Signal Based Surveillance, 2007. AVSS 2007. IEEE Conference on, vol. 3804, London, UK, 5-7 Sept. 2007, pp. 200-205.

[6] J. Yao and J.-M. Odobez, "Fast human detection from videos using covariance features," IDIAP Research Institute, Tech. Rep. 07-68, 2007.

[7] O. Tuzel, F. Porikli, and P. Meer, "Human detection via classification on riemannian manifolds," in CVPR, 2007.

[8] IST FP6-027231, "CARETAKER: Content Analysis and REtrieval Technologies to Apply Knowledge Extraction to massive Recording," 2006-2008, http://www.ist-caretaker.org/.

[9] G. Saporta, Probabilités, analyse des données et Statistique. Technip, 2006. 